\title{
Retroperitoneal Cystic Leiomyoma Masquerading as Ovarian Cyst, Presenting as Huge Incisional Hernia
}

\author{
Keyur Bhatt, Dhaval Mangukiya, Ajay Seth \\ Department of GI-HPB and Cancer Surgery, SIDS Hospital \& Research Centre, Surat, Gujarat, India.
}

\author{
Corresponding Author: \\ Dr. Keyur Bhatt \\ Email: drkeyurbhatt@gmail.com
}

This is an Open Access article distributed under the terms of the Creative Commons Attribution License (creativecommons.org/ licenses/by/3.0).

Published

\begin{abstract}
Background: Giant retroperitoneal cysts are uncommon, with an estimated incidence of $1 / 5750$ to $1 / 250,000$. Degenerative changes of leiomyoma may lead to complex presentation resulting in misdiagnosis. The final diagnosis can be made either during surgery or histologically. The most prevalent mesenchymal tumors at this site are of a lipomatous, myogenic or neuroectodermal nature. Case Report: We have a case of large leiomyoma of retroperitoneum with cystic degeneration confirmed on immunehistochemistry, which presented to us as right iliac fossa herniation masquerading as a huge right ovarian cyst. Conclusion: The retroperitoneal leiomyoma should be considered in the differential diagnosis of a giant cystic mass in abdomen.
\end{abstract}

Keywords: Leiomyoma, Lipoma, Neoplasms, Ovarian Cysts, Retroperitoneal Space.

\section{Introduction}

Retroperitoneal cysts are uncommon with an estimated incidence of $1 / 5750$ to $1 / 250,000$ [1]. Approximately one third of patients with retroperitoneal cysts are asymptomatic and found incidentally. The relative paucity of vital structures and the abundance of loose connective tissue in this area, results in late clinical presentation of space occupying lesions. Symptoms tend to be related to compression/invasion of adjacent structures. Malignant tumors of the retroperitoneum are roughly four times more frequent than benign lesions, in sharp contrast to neoplastic disease occurring elsewhere in the body [1-5].

\section{Case Report}

A 32 year-old woman was admitted with right sided abdominal out-pouching and pain in lower abdomen. She did not have bowel or bladder functional abnormality, fever, loss of appetite or weight loss. She had noticed the development of swelling for last five years, which slowly grew to current size. She had undergone right inguinal hernia surgery 18 years ago. On examination vitals were normal. The examination of abdomen revealed a mobile, soft, non-tender and nonreducible lump palpable in right iliac fosse of approximately $15 \times 16 \mathrm{~cm}$ in size, with stretched scar over it [Fig.1]. Ultrasonography of abdomen suggested a cystic lesion in right iliac fosse with intra and extra-abdominal components $(20 \times 16 \mathrm{~cm}$ outside abdomen and $23 \times 16 \mathrm{~cm}$ intra-abdominally extending from pelvic region to inferior surface of liver). All blood investigations were within normal limits.

Contrast enhanced computed tomography (CECT) scan of the abdomen revealed a cystic mass lesion measuring $20 \mathrm{~cm}$ in the right hepatorenal space reaching upto pelvis and out-pouching from right deep inguinal ring as hernia with $23 \times 16 \mathrm{~cm}$ lesion outside abdominal cavity. Mass was not involving any viscera of abdomen. Right ovary could not be identified separately from the mass. During surgery it was found to have large dumbbell 
shaped lesion arising from right retroperitoneum, herniating from the deep inguinal ring, cystic in nature, pushing entire peritoneum upwards and medially. Lesion was removed in toto with its walls and abdominal wall was repaired in layers with non-absorbable sutures.

Patient was discharged uneventfully on post-operative day four. The tumor on microscopic examination was composed of areas with different cellularity containing spindle cells. The stroma showed focal fibrous appearances with extensive edema and myxoid changes with scanty mitosis. Over all findings were suggestive of spindle cell neoplasm with extensive cystic degeneration. On immunohistochemistry, the tumor was diffusely positive for SMA, H-caldesmon and desmin which is suggestive of leiomyoma with cystic degeneration.

\section{Discussion}

Leiomyoma can outgrow their blood supply on enlargement resulting in various types of degeneration, such as hyaline, cystic or myxoid degeneration and dystrophic calcification [4]. Hyalinization is the most common type of degeneration, occurring in upto $60 \%$ of cases. Cystic degeneration, observed in about $4 \%$ of leiomyoma, may be considered extreme sequelae of edema [2]. The potential for retroperitoneal leiomyoma to grow to an extreme size before causing symptoms is quite remarkable. The atypical appearances that follow degenerative changes can cause confusion in diagnosis. Leiomyomas have been misdiagnosed as adenomyosis, hematometra, lymphangiomas and ovarian masses. The preferred imaging modality for the initial evaluation is ultrasonography. The relative echogenicity of leiomyoma's depends on the ratio of fibrous tissue to smooth muscle, the extent of degeneration and the presence of dystrophic calcification [3]. CECT is ideal for assessing retroperitoneal cysts (RPC) because it provides discrete sectional images of the organs and retroperitoneal compartments. Based on

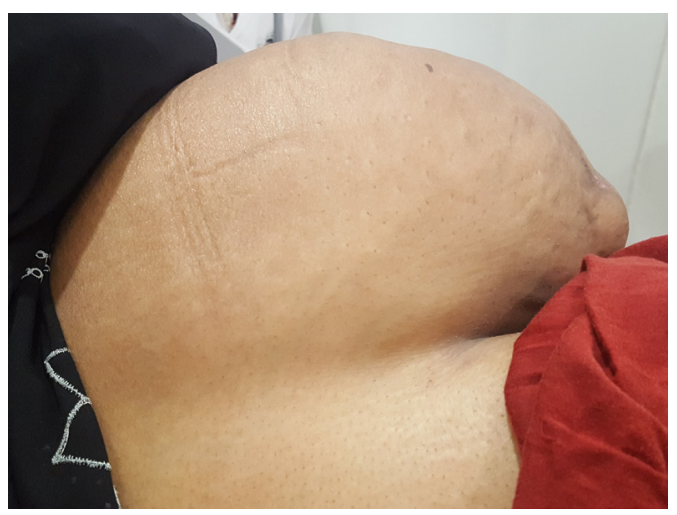

Fig.1: Clinical presentation of retroperitoneal cystic leiomyoma.

embryologic origin and histological differentiation, RPCs are classified into: urogenital; mesocolic; cysts arising in cell inclusions; traumatic; parasitic and lymphatic [6-8]. They have no pedicle and no connections apart from the areolar tissues with the surrounding structures. Differential diagnosis of retroperitoneal cysts in females includes: cystic mesothelioma, cystic hematoma, cystic teratoma, lymphangioma, pseudomyxoma peritonei, epidermoid cyst, tail gut cyst, mesenteric cyst, pseudocyst urinoma, lymphocele, mullerian cyst, vulval cyst, paraovarian cyst, vaginal cyst paraurethral cyst and mucinous cystadenoma.

There are no pathognomonic signs or symptoms for RPCs. Two thirds of patients present with an abdominal mass or chronic abdominal symptoms. Other symptoms include back pain, referred pain to the lower limbs, edema of the lower limbs, weight loss or fever [6]. Our patient had dumble shaped cystic lesion with intra and extra abdominal components. The cyst was almost occupying entire right retroperitoneum extending from pelvis to inferior surface of liver. Its pathogenesis was not known as the cyst did not have any epithelial lining. The diagnosis could only be confirmed after IHC examination.

\section{Conclusion}

Cysts arising within the retroperitoneum outside major organs are very rare. CT may help diagnose 
these lesions, but surgery remains the keystone in determining the diagnosis. This case is rare and educational as it presented as an unusually huge cyst with hernia masquerading ovarian cyst and finally turning out to be leiomyoma.

Contributors: KB: manuscript writing, patient management; DM: manuscript editing, patient management; AS: critical inputs into the manuscript. KB will act as a study guarantor. All authors approved the final version of this manuscript.

Funding: None; Competing interests: None stated.

\section{References}

1. Guile M, Fagan M, Simopolous A, Ellerkman M. Retroperitoneal cyst of Mullerian origin: A case report and review of the literature. J of Pelvic Medicine and Surgery. 2007;13(3):149-152.

2. Mayer DP, Shipilov V. Ultrasonography and magnetic resonance imaging of uterine fibroids. Obstet Gynecol Clin North Am. 1995;22:667-725.

3. Wladimiroff J. Uterine fibroids. In: Ultrasound in obstetrics and gynaecology. Elsevier; 2009. pp.303-306.

4. Preayson RA, Hart WR. Pathologic considerations of uterine smooth muscle tumors. Obstet Gynecol Clin North Am. 1995;22:637-657.

5. Handfield-Jones R. Retroperitoneal cysts, their pathology, diagnosis and treatment. British J Surg. 1942;12:119-134.

6. Konishi E, Nakashima Yi, Iwasaki T. Immunohistochemical analysis of retroperitoneal Mullerian cyst. Human Pathology. 2003;34(2):194-198.

7. Yang D, Jung D, Kim H, Kang H, Kim S, Kim J, Hwang H. Retroperitoneal cystic masses: CT, clinical and pathological findings and literature review. Radiographics. 2004;25(5):1353-1365.

8. Kurtz R, Heimann T, Beck R, Holt J. Mesenteric and retroperitoneal cysts. Ann Surg. 1986;203(1):109-112. 